Pacific

Journal of

Mathematics

\title{
A THEOREM OF MEGLIN AND WALDSPURGER FOR COVERING GROUPS
}

ShIV PRAKASH PATEL 


\title{
A THEOREM OF MOEGLIN AND WALDSPURGER FOR COVERING GROUPS
}

\author{
ShIV PRAKASH PATEL
}

\begin{abstract}
Let $E$ be a nonarchimedean local field of characteristic zero and residual characteristic $p$. Let $G$ be a connected reductive group defined over $E$ and $\pi$ an irreducible admissible representation of $G(E)$. A result of $C$. Moglin and J.-L. Waldspurger (for $p \neq 2$ ) and S. Varma (for $p=2$ ) states that the leading coefficient in the character expansion of $\pi$ at the identity element of $G(E)$ gives the dimension of a certain space of degenerate Whittaker forms. In this paper we generalize this result of Moglin and Waldspurger to the setting of covering groups of $G(E)$.
\end{abstract}

\section{Introduction}

Let $E$ be a nonarchimedean local field of characteristic zero, $\boldsymbol{G}$ a connected split reductive group defined over $E$, and $G=\boldsymbol{G}(E)$. Let $\mathfrak{g}=\operatorname{Lie}(\boldsymbol{G})$ be the Lie algebra of $\boldsymbol{G}$ and $\mathfrak{g}=\mathfrak{g}(E)$. Let $(\pi, W)$ be an irreducible admissible representation of $G$. A theorem of F. Rodier [1975] relates the dimension of the space of nondegenerate Whittaker forms of $\pi$ to coefficients in the character expansion of $\pi$ around the identity. More precisely, Rodier proved that if the residual characteristic of $E$ is large enough and the group $\boldsymbol{G}$ is split then the dimension of any space of nondegenerate Whittaker functionals for $(\pi, W)$ equals the coefficient in the character expansion of $\pi$ at the identity corresponding to an appropriate maximal nilpotent orbit in the Lie algebra $\mathfrak{g}$. Rodier proved his theorem assuming that the residual characteristic of $E$ is greater than a constant which depends only on the root datum of $\boldsymbol{G}$. C. Mœglin and J.-L. Waldspurger [1987] generalized this theorem of Rodier in several directions: in particular, they proved the result for fields $E$ whose residual characteristic is odd, and they removed the assumption that $\boldsymbol{G}$ is split. The Moglin-Waldspurger theorem is a more precise statement about the coefficients appearing in the character expansion around the identity and certain spaces of "degenerate" Whittaker forms. In [Varma 2014], this theorem has been proved for fields with even residual characteristic by modifying certain constructions in [Mœglin and Waldspurger 1987] (see the remark at the end of the introduction). So the Mœglin-Waldspurger theorem is

MSC2010: primary 22E50; secondary 11F70, 11S37.

Keywords: covering groups, character expansion, degenerate Whittaker forms. 
true for all connected reductive groups without any restriction on the residual characteristic of the field $E$. We now recall the theorem. To state it we need to introduce some notation. Let $Y$ be a nilpotent element in $\mathfrak{g}$ and suppose $\varphi: \mathbb{G}_{m} \rightarrow \boldsymbol{G}$ is a one-parameter subgroup satisfying

$$
\operatorname{Ad}(\varphi(t)) Y=t^{-2} Y .
$$

Associated to such a pair $(Y, \varphi)$ one can define a certain space $\mathcal{W}_{(Y, \varphi)}$, called the space of degenerate Whittaker forms of $(\pi, W)$ relative to $(Y, \varphi)$ (see Section 4 for the definition).

Define $\mathcal{N}_{\mathrm{Wh}}(\pi)$ to be the set of nilpotent orbits 0 of $\mathfrak{g}$ for which there exists an element $Y \in \mathcal{O}$ and a $\varphi$ satisfying (1) such that the space $\mathscr{W}_{(Y, \varphi)}$ of degenerate Whittaker forms relative to the pair $(Y, \varphi)$ is nonzero.

Recall that the character expansion of $(\pi, W)$ around the identity is a sum $\sum_{\mathscr{O}} c_{\mathscr{O}} \widehat{\mu_{\mathscr{O}}}$, where 0 varies over the set of nilpotent orbits of $\mathfrak{g}, c_{\mathscr{O}} \in \mathbb{C}$ and $\widehat{\mu_{\overparen{O}}}$ is the Fourier transform of a suitably chosen measure $\mu_{\mathscr{O}}$ on $\mathcal{O}$. One defines $\mathcal{N}_{\text {tr }}(\pi)$ to be the set of nilpotent orbits 0 of $\mathfrak{g}$ such that the corresponding coefficient $c_{\mathbb{O}}$ in the character expansion of $\pi$ around the identity is nonzero.

We have the standard partial order on the set of nilpotent orbits in $\mathfrak{g}: \mathrm{O}_{1} \leq \mathrm{O}_{2}$ if $\mathcal{O}_{1} \subset \overline{O_{2}}$. Let $\operatorname{Max} \mathcal{N}_{\text {Wh }}(\pi)$ and $\operatorname{Max} \mathcal{N}_{\text {tr }}(\pi)$ denote the sets of maximal elements in $\mathcal{N}_{\text {Wh }}(\pi)$ and $\mathcal{N}_{\text {tr }}(\pi)$, respectively, with respect to this partial order.

Theorem 1 [Mœglin and Waldspurger 1987, Chapter I]. Let $\boldsymbol{G}$ be a connected reductive group defined over $E$. Let $\pi$ be an irreducible admissible representation of $G=\boldsymbol{G}(E)$. Then

$$
\operatorname{Max} \mathcal{N}_{\mathrm{Wh}}(\pi)=\operatorname{Max} \mathcal{N}_{\text {tr }}(\pi) .
$$

Moreover, if $\mathcal{O}$ is an element in either of these sets, then for any $(Y, \varphi)$ as above with $Y \in \mathbb{O}$ we have

$$
c_{\mathscr{C}}=\operatorname{dim} \mathcal{W}_{(Y, \varphi)} .
$$

If one considers the case of the pair $(Y, \varphi)$ with $Y$ a regular nilpotent element, this theorem specializes to Rodier's theorem.

In this paper we generalize the Mœglin-Waldspurger theorem to the setting of a covering group $\tilde{G}$ of $G$. Let $\mu_{r}$ be the group of $r$-th roots of unity in $\mathbb{C}^{\times}$. An $r$-fold covering group $\tilde{G}$ of $G$ is a central extension of locally compact groups by $\mu_{r}:=\left\{z \in \mathbb{C}: z^{r}=1\right\}$ giving rise to the short exact sequence

$$
1 \longrightarrow \mu_{r} \longrightarrow \tilde{G} \longrightarrow G \longrightarrow 1 \text {. }
$$

The representations of $\tilde{G}$ on which $\mu_{r}$ acts by the natural embedding $\mu_{r} \hookrightarrow \mathbb{C}^{\times}$are called genuine representations. The definition of the space of degenerate Whittaker forms of a representation of $G$ involves only unipotent groups. Since the covering 
$\tilde{G} \rightarrow G$ splits over any unipotent subgroup of $G$ in a unique way (see [Mœglin and Waldspurger 1995]) this makes it possible to define the space of degenerate Whittaker forms for any genuine smooth representation $(\pi, W)$ of $\tilde{G}$. In particular, it makes sense to talk of the set $\mathcal{N}_{\text {Wh }}(\pi)$.

The existence of the character expansion of an admissible genuine representation $(\pi, W)$ of $\tilde{G}$ has been proved by Wen-Wei Li [2012]. At the identity, the HarishChandra-Howe character expansion of an irreducible genuine representation has the same form, and therefore we have $\mathcal{N}_{\text {tr }}(\pi)$. This makes it possible to have an analogue of Theorem 1 in the setting of covering groups. The main aim of this paper is to prove the following.

Theorem 2. Let $\pi$ be an irreducible admissible genuine representation of $\tilde{G}$. Then

$$
\operatorname{Max} \mathcal{N}_{\mathrm{Wh}}(\pi)=\operatorname{Max} \mathcal{N}_{\text {tr }}(\pi) .
$$

Moreover, if 0 is an element in either of these sets, then for any $(Y, \varphi)$ as above with $Y \in \mathbb{O}$ we have

$$
c_{\overparen{O}}=\operatorname{dim} \mathcal{W}_{(Y, \varphi)} .
$$

We will use the results in [Mœglin and Waldspurger 1987], and to accommodate the even residual characteristic case we follow [Varma 2014]. Let us describe some ideas involved in the proof. Let $Y$ be a nilpotent element in $\mathfrak{g}$ and $\varphi$ a one-parameter subgroup as above. Let $\mathfrak{g}_{i}$ be the eigenspace of weight $i$ under the action of $\mathbb{G}_{m}$ on $\mathfrak{g}$ via $\operatorname{Ad} \circ \varphi$. One can attach a parabolic subgroup $\boldsymbol{P}$ with unipotent radical $\boldsymbol{N}$ whose Lie algebras are $\mathfrak{p}:=\bigoplus_{i \geq 0} \mathfrak{g}_{i}$ and $\mathfrak{n}=\bigoplus_{i>0} \mathfrak{g}_{i}$ respectively. The oneparameter subgroup $\varphi$ also determines a parabolic subgroup $\boldsymbol{P}^{-}$opposite to $\boldsymbol{P}$ with Lie algebra $\mathfrak{p}^{-}=\bigoplus_{i \leq 0} \mathfrak{g}_{i}$. For simplicity, assume $\mathfrak{g}_{1}=0$ for the purpose of the introduction. Then $\mathfrak{n}=\bigoplus_{i \geq 2} \mathfrak{g}_{i}$ and $\chi: \gamma \mapsto \psi(B(Y, \log \gamma))$ defines a character of $N=N(E)$, where $B$ is an $\operatorname{Ad}(G)$-invariant nondegenerate symmetric bilinear form on $\mathfrak{g}$ and $\psi$ is an additive character of $E$. In this case (i.e., $\mathfrak{g}_{1}=0$ ), the space of degenerate Whittaker forms $\mathcal{W}_{(Y, \varphi)}$ is defined to be the twisted Jacquet module of $\pi$ with respect to $(N, \chi)$. In the case where $\mathfrak{g}_{1} \neq 0$, the definition of $\mathscr{W}_{(Y, \varphi)}$ needs to be appropriately modified (see Section 4).

On the other hand, to the pair $(Y, \varphi)$ one attaches certain open compact subgroups $G_{n}$ of $G$ for large $n$ and certain characters $\chi_{n}$ of $G_{n}$. One then proves that the covering $\tilde{G} \rightarrow G$ splits over $G_{n}$ for large $n$, so that the $G_{n}$ can be seen as subgroups of $\tilde{G}$ as well. Let $\varpi$ be a uniformizer of $E$. Let $t:=\varphi(\varpi)$ and $\tilde{t}$ be any lift of $t$ in $\tilde{G}$. It turns out that $\tilde{t}^{-n} G_{n} \tilde{t}^{n} \cap N$ becomes an "arbitrarily large" subgroup of $N$ and $\tilde{t}^{-n} G_{n} \tilde{t}^{n} \cap P^{-}$becomes an "arbitrarily small" subgroup of $P^{-}$, as $n$ becomes large. For large $n$, the characters $\chi_{n}$ have been so defined that the character $\chi_{n}^{\prime}:=\chi_{n} \circ \operatorname{Int}\left(\tilde{t}^{n}\right)$ restricted to $\tilde{t}^{-n} G_{n} \tilde{t}^{n} \cap N$ agrees with $\chi$. Using the Harish-Chandra-Howe character expansion, one proves that the dimension of the 
$\left(G_{n}, \chi_{n}\right)$-isotypic component of $W$ is equal to $c_{\mathscr{C}}$ for large enough $n$, where $\mathbb{O}$ is the nilpotent orbit of $Y$ in $\mathfrak{g}$. Finally, one proves that there is a natural isomorphism between the $\left(\tilde{t}^{-n} G_{n} \tilde{t}^{n}, \chi_{n} \circ \operatorname{Int}\left(\tilde{t}^{n}\right)\right)$-isotypic component of $W$ and $\mathcal{W}_{(Y, \varphi)}$.

Remark 3. The definition of $\mathcal{W}_{(Y, \varphi)}$ (hence that of $\left.\mathcal{N}_{\mathrm{Wh}}(\pi)\right)$ depends on a choice of an additive character $\psi$ of $E$ and a choice of $\operatorname{Ad}(G)$-invariant nondegenerate bilinear form $B$ on $\mathfrak{g}$. On the other hand, in the character expansion, the $c_{\overparen{O}}$ (hence $\mathcal{N}_{\text {tr }}(\pi)$ ) depend on $\psi, B$, a measure on $G$ and a measure on $\mathfrak{g}$. However, by choosing a compatible measure on $G$ and $\mathfrak{g}$ via the exponential map, one gets rid of the dependency of $c_{\mathscr{O}}$ on these measures, and therefore the $c_{\overparen{O}}$ depend only on $\psi$ and $B$. For more detailed discussion about how our results depend on $B$ and $\psi$, see Remark 4 in [Varma 2014].

Remark 4. One aspect in Varma's proof for $p=2$ which does not obviously generalize from the proof for $p \neq 2$ is the prescription of the character $\chi_{n}$ of $G_{n}$ given in [Mœglin and Waldspurger 1987], which is due to the somewhat bad behavior of the Campbell-Hausdorff formula in the $p=2$ case. Using Kirillov's theory of compact $p$-adic groups, Varma prescribed a $\chi_{n}$ (although not unique) which will serve our purpose. On the other hand, the definition of degenerate Whittaker forms of $W$ has also been modified by Varma to accommodate the case $p=2$.

Although the methods used in the paper are not new and heavily depend on the proofs in the linear case, the result is useful in the study of representation theory of covering groups. The author himself has made use of this result in his thesis, where he attempts to generalize a result of D. Prasad [1992] in the setting of covering groups, namely, in the harmonic analysis relating the pairs $\left(\mathrm{GL}_{2}(E)^{\sim}, \mathrm{GL}_{2}(F)\right)$ and $\left(\mathrm{GL}_{2}(E)^{\sim}, D_{F}^{\times}\right)$, where $E / F$ is a quadratic extension of nonarchimedean local field, $D_{F}$ is the quaternion division algebra with center $F$ and $\mathrm{GL}_{2}(E)^{\sim}$ is a certain twofold cover of $\mathrm{GL}_{2}(E)$.

Let us briefly give an outline of the organization of the paper. In Section 2, we recall the definition of the subgroups $G_{n}$ and state some properties of the character $\chi_{n}$. In Section 3, we recall splitting of the covering groups over $G_{n}$ and describe an appropriate choice of the splitting over the subgroup $G_{n}$ for large $n$. In Section 4 we give the definition of the space of degenerate Whittaker forms and describe important setup to prove the main theorem. In Section 5, we transfer some results from linear groups to covering groups in a few lemmas, and, based on these lemmas, we prove the main theorem.

\section{Subgroups $G_{n}$ and characters $\chi_{n}$}

In this section, we recall a certain sequence of subgroups $G_{n}$ of $G$, which form a basis of neighborhoods at the identity, and certain characters $\chi_{n}: G_{n} \rightarrow \mathbb{C}^{\times}$. The objects involved in this section were defined for linear groups in [Mœglin and 
Waldspurger 1987; Varma 2014], and we will lift them to covering groups in a suitable way in Section 3 and work with these lifts in this paper.

Let $\mathfrak{O}_{E}$ denote the ring of integers in $E$. We fix an additive character $\psi$ of $E$ with conductor $\mathfrak{O}_{E}$. Fix an $\operatorname{Ad}(G)$-invariant nondegenerate symmetric bilinear form $B: \mathfrak{g} \times \mathfrak{g} \rightarrow E$.

Let $Y \in \mathfrak{g}$ be nilpotent. Choose a one-parameter subgroup $\varphi: \mathbb{G}_{m} \rightarrow \boldsymbol{G}$ satisfying

$$
\operatorname{Ad}(\varphi(s)) Y=s^{-2} Y \quad \text { for all } s \in \mathbb{G}_{m} .
$$

For a given nilpotent element $Y$, the existence of such a $\varphi$ is known from the theory of $\mathfrak{s l}_{2}$-triplets, but there are examples which do not come from this theory.

For $i \in \mathbb{Z}$, define

$$
\mathfrak{g}_{i}=\left\{X \in \mathfrak{g}: \operatorname{Ad}(\varphi(s)) X=s^{i} X \text { for all } s \in \mathbb{G}_{m}\right\} .
$$

Set

$$
\mathfrak{n}:=\mathfrak{n}^{+}:=\bigoplus_{i>0} \mathfrak{g}_{i}, \quad \mathfrak{n}^{-}:=\bigoplus_{i<0} \mathfrak{g}_{i}, \quad \mathfrak{p}^{-}:=\bigoplus_{i \leq 0} \mathfrak{g}_{i}
$$

The parabolic subgroup $\boldsymbol{P}^{-}$of $\boldsymbol{G}$ stabilizing $\mathfrak{n}^{-}$has $\mathfrak{p}^{-}$as its Lie algebra. Let $\boldsymbol{N}=\boldsymbol{N}^{+}$be the unipotent subgroup of $\boldsymbol{G}$ having the Lie algebra $\mathfrak{n}$.

Let $G(Y)$ be the centralizer of $Y$ in $G$ and $Y^{\#}$ the centralizer of $Y$ in $\mathfrak{g}$. The $G$-orbit $\mathcal{O}_{Y}$ of $Y$ can be identified with $G / G(Y)$ and therefore its tangent space at $Y$ can be identified with $\mathfrak{g} / Y^{\#}$. Note that

$$
\begin{aligned}
Y^{\#} & =\{X \in \mathfrak{g}:[X, Y]=0\} \\
& =\{X \in \mathfrak{g}: B([X, Y], Z)=0 \text { for all } Z \in \mathfrak{g}\} \\
& =\{X \in \mathfrak{g}: B(Y,[X, Z])=0 \text { for all } Z \in \mathfrak{g}\} .
\end{aligned}
$$

The bilinear form $B$ induces a nondegenerate alternating form

$$
B_{Y}: \mathfrak{g} / Y^{\#} \times \mathfrak{g} / Y^{\#} \longrightarrow E
$$

defined by $B_{Y}\left(X_{1}, X_{2}\right)=B\left(Y,\left[X_{1}, X_{2}\right]\right)$.

Let $L \subset \mathfrak{g}$ be a lattice satisfying the following conditions:

(1) $[L, L] \subset L$;

(2) $L=\bigoplus_{i \in \mathbb{Z}} L_{i}$, where $L_{i}=L \cap \mathfrak{g}_{i}$;

(3) The lattice $L / L_{Y}$, where $L_{Y}=L \cap Y^{\#}$, is self-dual with respect to $B_{Y}$, i.e., $\left(L / L_{Y}\right)^{\perp}=L / L_{Y}$. (For any vector space $V$ with a nondegenerate bilinear form $B$ and a lattice $M$ in $V, M^{\perp}:=\left\{X \in V: B(X, Y) \in \mathfrak{O}_{E}\right.$ for all $\left.Y \in V\right\}$.)

A lattice $L$ satisfying these properties can be chosen by taking a suitable basis of all the $\mathfrak{g}_{i}$; see [Mœglin and Waldspurger 1987]. Now we summarize a few well-known 
properties of the exponential map, and use them to define subgroups $G_{n}$ and their Iwahori decompositions.

Lemma 5. (1) There exists a positive integer A such that exp is defined and injective on $\varpi^{A} L$, with inverse $\log$.

(2) The exponential map on $\varpi^{n} L$ is a homeomorphism onto its image $G_{n}:=$ $\exp \left(\varpi^{n} L\right)$, which is an open subgroup of $G$ for all $n \geq A$.

(3) Set $P_{n}^{-}=\exp \left(\varpi^{n} L \cap \mathfrak{p}^{-}\right)$and $N_{n}=\exp \left(\varpi^{n} L \cap \mathfrak{n}\right)$. Then we have an Iwahori factorization

$$
G_{n}=P_{n}^{-} N_{n}
$$

We will be working with a certain character $\chi_{n}$ of $G_{n}$, which we recall in the next lemma.

Lemma 6. For large values of $n$ there exists a character $\chi_{n}$ of $G_{n}$ whose restriction to $\exp \left(\left(Y^{\#} \cap \varpi^{n} L\right)+\varpi^{n+\mathrm{val} 2} L\right)$ coincides with $\gamma \mapsto \psi\left(B\left(\varpi^{-2 n} Y, \log \gamma\right)\right)$. If $P_{n}^{-}$ is as in Lemma 5, the character $\chi_{n}$ can be chosen so that

$$
\chi_{n}(p)=1 \text { for all } p \in P_{n}^{-} .
$$

For a proof of this lemma and other properties of this character $\chi_{n}$ see Lemma 5 in [Varma 2014].

Remark 7. If $p \neq 2$, then the map $\gamma \mapsto \psi\left(B\left(\varpi^{-2 n} Y, \log \gamma\right)\right)$ itself defines a character of $G_{n}$ for large $n$ and satisfies the properties stated in Lemma 6. But for $p=2$, there is more than one such character $\chi_{n}$; for more details see [Varma 2014].

\section{Covering groups}

Let $\mu_{r}$ be the group of $r$-th roots of unity in $\mathbb{C}$. Consider an $r$-fold covering $\tilde{G}$ of $G$. Recall that this is a central extension of locally compact groups of the group $G$ by $\mu_{r}$ giving rise to the short exact sequence

$$
1 \longrightarrow \mu_{r} \longrightarrow \tilde{G} \longrightarrow G \longrightarrow 1 \text {. }
$$

Lemma 8. (1) The covering $\tilde{G} \rightarrow G$ splits uniquely over any unipotent subgroup of $G$.

(2) For large enough $n$ the covering $\tilde{G} \rightarrow G$ splits over $G_{n}$. Moreover, there is a splitting $s$ of $\tilde{G} \rightarrow G$ restricted to $\bigcup_{g \in G} g G_{n} g^{-1}$ such that $s\left(h t h^{-1}\right)=$ $h s(t) h^{-1}$ for all $h \in G$.

Proof. (1) This is well known; see [Mœglin and Waldspurger 1995]. For a simpler proof in the case when $E$ has characteristic zero, see Section 2.2 of [Li 2014]. 
(2) Recall that the subgroups $G_{n}$ form a basis of neighborhoods of the identity. It is well known that the covering $\tilde{G} \rightarrow G$ splits over a neighborhood of the identity. Therefore, for large enough $n$, the covering splits over $G_{n}$. There is more than one possible splitting of the cover $\tilde{G} \rightarrow G$ over $G_{n}$. If a splitting is fixed, then any other splitting over $G_{n}$ will differ from the above splitting by a character $G_{n} \rightarrow \mu_{r}$.

Fix some $m$ such that the covering splits over $G_{m}=\exp \left(\varpi^{m} L\right)$. As mentioned above, any two splittings over the subgroup $G_{m}$ will differ by a character $G_{m} \rightarrow \mu_{r}$ and any such character is trivial over

$$
G_{m}^{r}:=\left\{g^{r}: g \in G_{m}\right\} .
$$

Hence all the possible splittings over $G_{m}$ agree on $G_{m}^{r}$. The subset $G_{m}^{r}$ is a subgroup of $G_{m}$ as it equals $\exp \left(r \cdot \varpi^{m} L\right)$. Let $g, h \in G$. We have

$$
g G_{m} g^{-1} \cap h G_{m} h^{-1} \supset g G_{m}^{r} g^{-1} \cap h G_{m}^{r} h^{-1} .
$$

This implies that any two splittings of $\tilde{G} \rightarrow G$ restricted to $g G_{m}^{r} g^{-1} \cap h G_{m}^{r} h^{-1}$, one coming from the restriction of a splitting of $\tilde{G} \rightarrow G$ over $g G_{m} g^{-1}$ and the other coming from the restriction of a splitting over $h G_{m} h^{-1}$, are the same. Now choose $A^{\prime}$ so large such that $G_{n} \subset G_{m}^{r}$ for $n \geq A^{\prime}$. We fix the splitting of $G_{n}$ which comes from that of the restriction of $G_{m}^{r}$. This gives us a splitting over $\bigcup_{g \in G} g G_{n} g^{-1}$.

Using this splitting we get that the exponential map is defined from a small enough neighborhood of $\mathfrak{g}$ to $\tilde{G}$, namely the usual exponential map composed with this splitting, which one can use to define the character expansion of an irreducible admissible genuine representation $(\pi, W)$ of $\tilde{G}$, which was done in [Li 2012].

Remark 9. If $r$ is coprime to $p$, then as $G_{n}$ is a pro- $p$ group and $(r, p)=1$, there is no nontrivial character from $G_{n}$ to $\mu_{r}$. In that situation, the splitting in the preceding lemma is unique.

From now onwards, for large enough $n$, we treat $G_{n}$ not only as a subgroup of $G$ but also as one of $\tilde{G}$, with the above specified splitting. In other words, for the covering group $\tilde{G}$ (as in the linear case) we have a sequence of pairs $\left(G_{n}, \chi_{n}\right)$ using the splitting specified above which satisfies the properties described in Section 2.

Definition 10. Let $H \subset G$ be an open subgroup and $s: H \hookrightarrow \tilde{G}$ be a splitting. Then for any $\phi \in C_{c}^{\infty}(G)$ with $\operatorname{supp}(\phi) \subset H$, define $\tilde{\phi}_{s} \in C_{c}^{\infty}(\tilde{G})$ by

$$
\tilde{\phi}_{s}(g):= \begin{cases}\phi\left(g^{\prime}\right) & \text { if } g=s\left(g^{\prime}\right) \in s(H), \\ 0 & \text { if } g \in \tilde{G} \backslash s(H) .\end{cases}
$$

Note that this definition depends upon the choice of splitting. Whenever the splitting is clear in the context or it has been fixed and there is no confusion we write just $\tilde{\phi}$ 
instead of $\tilde{\phi}_{s}$ and $H$ for $s(H)$. Recall that the convolution $\phi * \phi^{\prime}$ for $\phi, \phi^{\prime} \in C_{c}^{\infty}(G)$ is defined by

$$
\phi * \phi^{\prime}(x)=\int_{G} \phi\left(x y^{-1}\right) \phi^{\prime}(y) d y .
$$

Observe that

$$
\operatorname{supp}\left(\phi * \phi^{\prime}\right) \subset \operatorname{supp}(\phi) \cdot \operatorname{supp}\left(\phi^{\prime}\right),
$$

which implies the lemma below.

Lemma 11. Let $H$ be an open subgroup of $G$ such that the covering $\tilde{G} \rightarrow G$ has a splitting over $H$, say, $s: H \hookrightarrow \tilde{G}$, satisfying $s(x y)=s(x) s(y)$ whenever $x$ and $y$ are in $H$. If $\phi, \phi^{\prime} \in C_{c}^{\infty}(G)$ are such that supp $(\phi)$ and supp $\left(\phi^{\prime}\right)$ are contained in $H$, then we have

$$
\widetilde{\phi * \phi^{\prime}}=\tilde{\phi} * \tilde{\phi}^{\prime} .
$$

\section{Degenerate Whittaker forms}

In this section we give the definition of degenerate Whittaker forms for a smooth genuine representation $\pi$ of $\tilde{G}$. This is an adaptation of Section I.7 of [Mœglin and Waldspurger 1987] and Section 5 of [Varma 2014].

Define

$$
N:=\exp \mathfrak{n}=\exp \bigoplus_{i \geq 1} \mathfrak{g}_{i}, \quad N^{2}:=\exp \bigoplus_{i \geq 2} \mathfrak{g}_{i}, \quad \text { and } N^{\prime}:=\exp \left(\mathfrak{g}_{1} \cap Y^{\#}\right) N^{2} .
$$

It is easy to see that $N^{2}$ and $N^{\prime}$ are normal subgroups of $N$. Let $H$ be the Heisenberg group defined with $\mathfrak{g}_{1} /\left(\mathfrak{g}_{1} \cap Y^{\#}\right) \times E$ as underlying set using the symplectic form induced by $B_{Y}$, i.e., for $X, Z \in \mathfrak{g}_{1} /\left(\mathfrak{g}_{1} \cap Y^{\#}\right)$ and $a, b \in E$,

$$
(X, a)(Z, b)=\left(X+Z, a+b+\frac{1}{2} B_{Y}(X, Z)\right) .
$$

Consider the map $N \rightarrow H$ given by

$$
\exp X \mapsto(\bar{X}, B(Y, X)),
$$

where $\bar{X}$ is the image of the $\mathfrak{g}_{1}$ component of $X$ in $\mathfrak{g}_{1} /\left(\mathfrak{g}_{1} \cap Y^{\#}\right)$. The CampbellHausdorff formula implies that this map is a homomorphism with kernel

$$
N^{\prime \prime}=\left\{n \in N^{\prime}: B(Y, \log n)=0\right\} .
$$

Let $\chi: N^{\prime} \rightarrow \mathbb{C}^{\times}$be defined by $\gamma \mapsto \psi \circ B(Y, \log \gamma)$. Note that

$$
\gamma \mapsto B(Y, \log \gamma) \in E \cong\{0\} \times E \subset H
$$

induces an isomorphism $N^{\prime} / N^{\prime \prime} \cong E$. 
We note that the cover $\tilde{G} \rightarrow G$ splits uniquely over the subgroups $N, N^{\prime}$ and $N^{\prime \prime}$. We denote the images of these splittings inside $\tilde{G}$ by the same letters. For a smooth genuine representation $(\pi, W)$ of $\tilde{G}$ we define

$$
N_{\chi}^{2} W=\left\{\pi(n) w-\chi(n) w: w \in W, n \in N^{2}\right\}
$$

and

$$
N_{\chi}^{\prime} W=\left\{\pi(n) w-\chi(n) w: w \in W, n \in N^{\prime}\right\} .
$$

Note that $N$ normalizes $\chi$, therefore $H=N / N^{\prime \prime}$ acts on $W / N_{\chi}^{\prime} W$ in a natural way. This action restricts to $N^{\prime} / N^{\prime \prime}$ (the center of $N / N^{\prime \prime}$ ) as multiplication by the character $\chi$. Let $\mathscr{Y}$ be the unique irreducible representation of the Heisenberg group $H$ with central character $\chi$.

Definition 12. Define the space of degenerate Whittaker forms for $(\pi, W)$ associated to $(Y, \varphi)$ to be

$$
\mathscr{W}:=\operatorname{Hom}_{H}\left(\mathscr{Y}, W / N_{\chi}^{\prime} W\right) .
$$

Remark 13. If $\mathfrak{g}_{1}=0$, then $N=N^{\prime}=N^{2}$. In this case, $\mathscr{W} \cong W / N_{\chi} W$ is the $(N, \chi)$-twisted Jacquet functor.

Definition 14. For a smooth representation $(\pi, W)$ of $\tilde{G}$, define $\mathcal{N}_{W h}(\pi)$ to be the set of nilpotent orbits $\mathbb{O}$ of $\mathfrak{g}$ such that there exist $Y \in \mathbb{O}$ and $\varphi$ as in (3), such that the space of degenerate Whittaker forms for $\pi$ associated to $(Y, \varphi)$ is nonzero.

As $\mathfrak{g}_{1} / \mathfrak{g}_{1} \cap Y^{\#}$ is a symplectic vector space and $L / L_{Y}$ is self-dual, it follows that $L_{H}:=\left(L \cap \mathfrak{g}_{1}\right) /\left(L \cap \mathfrak{g}_{1} \cap Y^{\#}\right)$ is a self-dual lattice in the symplectic vector space $H / Z(H) \cong \mathfrak{g}_{1} /\left(\mathfrak{g}_{1} \cap Y^{\#}\right)$.

Recall the definition (4) of the Heisenberg group $H$. Since $\psi$ is trivial on $\mathfrak{O}_{E}$, it follows that one can extend the character $\psi$ of $E \cong Z(H)$ to a character of the inverse image of $2 L_{H}$ under $H \rightarrow \mathfrak{g}_{1} /\left(\mathfrak{g}_{1} \cap Y^{\#}\right)$ by defining it to be trivial on $2 L_{H} \times\{0\} \subset H$. From Lemma 4 in [Varma 2014], this character can be extended to a character $\tilde{\chi}$ on the inverse image $H_{0}$ of $L_{H}$ under the natural map $H \rightarrow \mathfrak{g}_{1} /\left(\mathfrak{g}_{1} \cap Y^{\#}\right)$.

Remark 15. There are one-parameter subgroups $\varphi$ which do not arise from $\mathfrak{s l}_{2}-$ triplets. If $\varphi$ arises from an $\mathfrak{s l}_{2}$-triplet, then it is easy to see that $Y^{\#} \subset \bigoplus_{i \leq 0} \mathfrak{g}_{i}$. In particular, we have $\mathfrak{g}_{1} \cap Y^{\#}=\{0\}$ and hence the Heisenberg group $H$ coincides with $\mathfrak{g}_{1} \times E$.

Then, by Chapter 2, Section I.3 of [Mœglin et al. 1987], one knows that $\mathscr{Y}=$ $\operatorname{ind}_{H_{0}}^{H} \tilde{\chi}$ (induction with compact support). Since $H_{0}$ is an open subgroup of the locally profinite group $H$, we have the Frobenius reciprocity law

$$
\operatorname{Hom}_{H}(\mathscr{Y}, \tau)=\operatorname{Hom}_{H}\left(\operatorname{ind}_{H_{0}}^{H} \tilde{\chi}, \tau\right)=\operatorname{Hom}_{H_{0}}\left(\tilde{\chi},\left.\tau\right|_{H_{0}}\right)
$$

for any smooth representation $\tau$ of $H$. Thus, in the category of representations of $N$ on which $N^{\prime}$ acts via the character $\chi$, the functor $\operatorname{Hom}_{H}(\mathscr{Y},-)$ amounts to 
taking the $\left.\tilde{\chi}\right|_{H_{0}}$-isotypic component. Since $H_{0}$ is compact modulo the center, this functor is exact. Thus, we have

$$
\mathscr{W}=\operatorname{Hom}_{H}\left(\mathscr{Y}, W / N_{\chi}^{\prime} W\right) \cong\left(W / N_{\chi}^{\prime} W\right)^{\left(H_{0}, \tilde{\chi}\right)} .
$$

where $\left(W / N_{\chi}^{\prime} W\right)^{\left(H_{0}, \tilde{\chi}\right)}$ denotes the $\left(H_{0}, \tilde{\chi}\right)$-isotypic component of $W / N_{\chi}^{\prime} W$.

Recall that we have defined certain characters $\chi_{n}$ in Section 2 and now we have a character $\tilde{\chi}$. We need to choose them in a compatible way. First we fix a character $\tilde{\chi}$ and consider it as a character of $\exp \left(\mathfrak{g}_{1} \cap L\right) N^{\prime}$ in the obvious way $\left(\operatorname{as} \exp \left(\mathfrak{g}_{1} \cap L\right) N^{\prime}\right.$ is the inverse image of $H_{0}$ under $N \rightarrow H$ ). Let $t:=\varphi(\varpi) \in G$. Let $\tilde{t} \in \tilde{G}$ be any lift of $t$ in $\tilde{G}$. Let

$$
G_{n}^{\prime}=\operatorname{Int}\left(\tilde{t}^{-n}\right)\left(G_{n}\right), \quad P_{n}^{\prime}=\operatorname{Int}\left(\tilde{t}^{-n}\right)\left(P_{n}^{-}\right) \quad \text { and } \quad V_{n}^{\prime}=\operatorname{Int}\left(\tilde{t}^{-n}\right)\left(N_{n}\right) .
$$

It can be easily verified that $V_{n}^{\prime}$ contains $\exp \left(\mathfrak{g}_{1} \cap L\right)$. We also have $V_{n}^{\prime} \subset V_{m}^{\prime}$ for large $m, n$ with $n \leq m$. Moreover,

$$
\exp \left(\mathfrak{g}_{1} \cap L\right) N^{2}=\bigcup_{n \geq 0} V_{n}^{\prime} .
$$

It can also be verified easily that $\tilde{\chi} \circ \operatorname{Int}\left(\tilde{t}^{-n}\right)$ restricts to a character of $N_{n}$ that extends the character on $N_{n+\text { val } 2} N_{n}^{\prime}$ given by $\gamma \mapsto \psi\left(B\left(\varpi^{-2 n} Y, \log \gamma\right)\right)$. Now define

$$
\chi_{n}(p v)=\tilde{\chi}\left(\tilde{t}^{-n} v \tilde{t}^{n}\right) \quad \text { for all } p \in P_{n}^{-} \text {and all } v \in V_{n}^{\prime} .
$$

Lemma 16 [Varma 2014, Lemma 6]. Let $\chi_{n}$ be as defined in (5). Then $\chi_{n}$ is a character of $G_{n}$ and satisfies the properties stated in Lemma 6.

Define a character $\chi_{n}^{\prime}$ on $G_{n}^{\prime}$ by

$$
\chi_{n}^{\prime}:=\chi_{n} \circ \operatorname{Int}\left(\tilde{t}^{n}\right) .
$$

Remark 17. The characters $\chi_{n}$ have been defined so that the $\chi_{n}^{\prime}$ agree with $\chi$ on the intersection of their domains, namely, for large $n$ we have

$$
\left.\chi_{n}^{\prime}\right|_{V_{n}^{\prime}}=\left.\tilde{\chi}\right|_{V_{n}^{\prime}} .
$$

In particular, $\left.\chi_{n}^{\prime}\right|_{\exp \left(L \cap \mathfrak{g}_{1}\right)}=\left.\tilde{\chi}\right|_{\exp \left(L \cap \mathfrak{g}_{1}\right)}$. One can also see that $\chi_{n}^{\prime}$ and $\chi_{m}^{\prime}$ (for large $n, m$ ) agree on $G_{n}^{\prime} \cap G_{m}^{\prime}$, because they agree on $V_{n}^{\prime} \cap V_{m}^{\prime}$ and also on $P_{n}^{\prime} \cap P_{m}^{\prime}$ (being trivial on it).

Set

$$
W_{n}:=\left\{w \in W: \pi(\gamma) w=\chi_{n}(\gamma) w \text { for all } \gamma \in G_{n}\right\}
$$

and

$$
W_{n}^{\prime}:=\left\{w \in W: \pi(\gamma) w=\chi_{n}^{\prime}(\gamma) w \text { for all } \gamma \in G_{n}^{\prime}\right\}=\pi\left(\tilde{t}^{-n}\right) W_{n}
$$


For large $m, n$, define the map $I_{n, m}^{\prime}: W_{n}^{\prime} \rightarrow W_{m}^{\prime}$ by

$$
I_{n, m}^{\prime}(w)=\int_{G_{m}^{\prime}} \chi_{m}^{\prime}\left(\gamma^{-1}\right) \pi(\gamma) w d \gamma
$$

Let $m, n$ be large with $m>n$. Since $\chi_{n}^{\prime}$ is trivial on $P_{n}^{\prime} \supset P_{m}^{\prime}$ and since $G_{m}^{\prime}=P_{m}^{\prime} V_{m}^{\prime}$, for a convenient choice of measures we have

$$
\begin{aligned}
I_{n, m}^{\prime}(w) & =\int_{V_{m}^{\prime}} \chi_{m}^{\prime}\left(x^{-1}\right) \pi(x) w d x \\
& =\int_{\exp \left(\mathfrak{g}_{1} \cap L\right)} \tilde{\chi}^{-1}(\exp X) \pi(\exp X) \int_{N^{2} \cap G_{m}^{\prime}} \chi\left(x^{-1}\right) \pi(x) w d x d X .
\end{aligned}
$$

Now using the fact that $\exp \left(\mathfrak{g}_{1} \cap L\right)$ lies in $G_{n}^{\prime}$ for large $n$ and that it normalizes the character $\left.\chi\right|_{N^{2}}$, we get

$$
I_{n, m}^{\prime}(w)=\int_{N^{2} \cap G_{m}^{\prime}} \chi\left(x^{-1}\right) \pi(x) w d x=\int_{N^{\prime} \cap G_{m}^{\prime}} \chi\left(x^{-1}\right) \pi(x) w d x .
$$

From this the following is clear for large $n, m$ with $m>n$ :

$$
I_{n, m}^{\prime}=I_{n+1, m}^{\prime} \circ I_{n, n+1}^{\prime} \text {. }
$$

For large $n$, this equation gives that $\operatorname{ker} I_{n, m}^{\prime} \subset \operatorname{ker} I_{n, p}^{\prime}$ for $n<m \leq p$. Set

$$
W_{n, \chi}^{\prime}:=\bigcup_{m>n} \operatorname{ker} I_{n, m}^{\prime}
$$

Recall that for any unipotent subgroup $U$, character $\chi: U \rightarrow \mathbb{C}^{\times}$and $w \in W$, we have that

$$
\int_{K} \chi(x)^{-1} \pi(x) w d x=0
$$

for some open compact subgroup $K$ of $U$ if and only if $w \in U_{\chi} W$, where $U_{\chi} W$ is the span of $\{\pi(u) w-\chi(u) w: u \in U, w \in W\}$. Thus we have $W_{n, \chi} \subset N_{\chi}^{2} W$ as well as $W_{n, \chi} \subset N_{\chi}^{\prime} W$, which gives the natural maps

$$
j_{n}: W_{n}^{\prime} / W_{n, \chi}^{\prime} \longrightarrow W / N_{\chi}^{2} W \quad \text { and } \quad j_{n}^{\prime}: W_{n}^{\prime} / W_{n, \chi}^{\prime} \longrightarrow W / N_{\chi}^{\prime} W,
$$

and these give the diagram

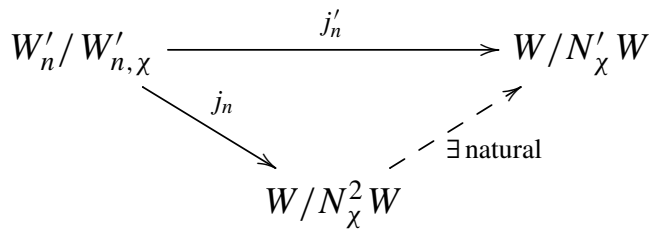


By the compatibility between $\chi_{n}^{\prime}$ and $\tilde{\chi}$, it is easy to see that the image of $j_{n}^{\prime}$ is contained in $\left(W / N_{\chi}^{\prime} W\right)^{\left(H_{0}, \tilde{\chi}\right)}$. Let $w \in W$ such that the image $\bar{w}$ of $w$ in $W / N_{\chi}^{\prime} W$ belongs to $\left(W / N_{\chi}^{\prime} W\right)^{\left(H_{0}, \tilde{\chi}\right)}$. For large $n, P_{n}^{\prime}$ acts trivially on $w$, as $(\pi, W)$ is smooth. Since $G_{n}^{\prime}=P_{n}^{\prime} V_{n}^{\prime}=V_{n}^{\prime} P_{n}^{\prime}$, the element

$$
\int_{V_{n}^{\prime}} \chi_{n}^{\prime}\left(x^{-1}\right) \pi(x) w d x
$$

belongs to $W_{n}^{\prime}$. As $\chi_{n}^{\prime}$ and $\chi$ are compatible, it can be seen that its image in $W / N_{\chi}^{\prime} W$ is $\bar{w}$. This gives us the following lemma.

Lemma 18. Let $(Y, \varphi)$ be arbitrary. Then any element of $\left(W / N_{\chi}^{\prime} W\right)^{\left(H_{0}, \chi\right)}$ belongs to $j_{n}^{\prime}\left(W_{n}^{\prime}\right)$ for all sufficiently large $n$. In particular, if $\mathcal{W} \neq 0$, then $W_{n}$ and $W_{n}^{\prime}$ are nonzero for large $n$.

\section{Main theorem}

Now recall that, by the work of Li [2012], the Harish-Chandra-Howe character expansion of an irreducible admissible genuine representation of $\tilde{G}$ at the identity element has an expression of the same form as that of an irreducible admissible representation of a linear group. The proof of the following lemma for a covering group follows verbatim that of Proposition I.11 in [Mœglin and Waldspurger 1987] and Proposition 1 in [Varma 2014].

Proposition 19. Let $\mathcal{W}$ be the space of degenerate Whittaker forms for $\pi$ with respect to a given $(Y, \varphi)$. If $\mathscr{W} \neq 0$, then there exists a nilpotent orbit 0 in $\mathcal{N}_{\operatorname{tr}}(\pi)$ such that $\mathrm{O}_{Y} \leq \mathbb{0}$, i.e., $Y \in \overline{\mathrm{O}}$.

Let the function $\phi_{n}: G \rightarrow \mathbb{C}$ be defined by

$$
\phi_{n}(\gamma)= \begin{cases}\chi_{n}\left(\gamma^{-1}\right) & \text { if } \gamma \in G_{n} \\ 0 & \text { otherwise }\end{cases}
$$

Consider the corresponding function $\tilde{\phi}_{n}: \tilde{G} \rightarrow \mathbb{C}$. Write the character expansion at the identity element as

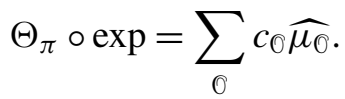

Choose $n$ large enough so that this expansion is valid over $G_{n}$, and then evaluate $\Theta_{\pi}$ at the function $\tilde{\phi}_{n}$. As $\pi\left(\tilde{\phi}_{n}\right)$ is a projection from $W$ to $W_{n}$, by definition we get $\Theta_{\pi}\left(\tilde{\phi}_{n}\right)=\operatorname{trace} \pi\left(\tilde{\phi}_{n}\right)=\operatorname{dim} W_{n}$. Now assume that $(Y, \varphi)$ is such that $O_{Y}$ is a

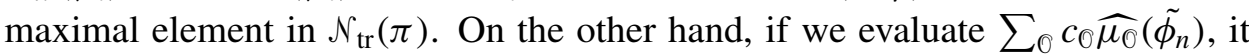
turns out that $\widehat{\mu_{\mathcal{O}}}\left(\tilde{\phi}_{n}\right)$ is zero unless $\widehat{O}=\hat{O}_{Y}$. In addition, if we fix a $G$-invariant measure on $O_{Y}$ as in I.8 of [Mœglin and Waldspurger 1987] (for more details about this invariant measure see Section 3 of [Varma 2014]), we get the following lemma. 
Lemma 20 (Lemma I.12 in [Mœglin and Waldspurger 1987] and Lemma 7 in [Varma 2014]). If $(Y, \varphi)$ is such that $\mathcal{O}_{Y}$ is a maximal element of $\mathcal{N}_{\text {tr }}(\pi)$. Then for large $n$,

$$
\operatorname{dim} W_{n}=c_{\mathscr{O}_{Y}} .
$$

In particular, the dimension of $W_{n}$ is finite and independent of $n$, for large $n$.

From Lemma 18 we know that every vector in $\mathcal{W}$ is in the image of $j_{n}^{\prime}$ for large $n$. In particular, if $W_{n}$ is finite-dimensional, we get that the map $j_{n}^{\prime}$ is surjective. Moreover, we have the following lemma, whose proof is verbatim that of Corollary I.14 in [Mœglin and Waldspurger 1987] and Lemma 8 in [Varma $2014]$ in the case of a linear group.

Lemma 21. Let $(Y, \varphi)$ be such that $O_{Y}$ is a maximal element of $\mathcal{N}_{\text {tr }}(\pi)$. Then for large $n$, the maps $j_{n}$ and $j_{n}^{\prime}$ are injections and the image of $j_{n}^{\prime}$ is $\left(W / N_{\chi}^{\prime} W\right)^{\left(H_{0}, \tilde{\chi}\right)}$.

Let $\phi_{n}^{\prime}: G \rightarrow \mathbb{C}$ be defined by

$$
\phi_{n}^{\prime}(\gamma)= \begin{cases}\chi_{n}^{\prime}\left(\gamma^{-1}\right) & \text { if } \gamma \in G_{n}^{\prime} \\ 0 & \text { otherwise }\end{cases}
$$

Consider the corresponding function $\tilde{\phi}_{n}^{\prime}: \tilde{G} \rightarrow \mathbb{C}$.

Lemma 22. Consider a pair $(Y, \varphi)$ such that $\mathbb{O}=O_{Y}$ is a maximal in $\mathcal{N}_{\text {tr }}(\pi)$. Then, for large enough $n$ :

(1) Let $\mathscr{Y}_{n} \subset G_{n+1}^{\prime} \cap G(Y)$ be a set of representatives for the $G_{n}^{\prime}$ double cosets in $G_{n}^{\prime}\left(G_{n+1} \cap G(Y)\right) G_{n}^{\prime}$. Then for large enough $n$,

$\tilde{\phi}_{n}^{\prime} * \tilde{\phi}_{n+1}^{\prime} * \tilde{\phi}_{n}^{\prime}(g)= \begin{cases}\lambda \cdot\left(\chi_{n}^{\prime}\right)^{-1}\left(h_{1} h_{2}\right) & \text { if } g=h_{1} y h_{2} \text { with } y \in \mathscr{Y}_{n}, h_{1}, h_{2} \in G_{n}^{\prime}, \\ 0 & \text { if } g \notin G_{n}^{\prime} \mathscr{Y}_{n} G_{n}^{\prime},\end{cases}$

where $\lambda=\operatorname{meas}\left(G_{n}^{\prime} \cap G_{n+1}^{\prime}\right) \operatorname{meas}\left(G_{n}^{\prime}\right)$.

(2) For large $n, I_{n, n+1}^{\prime}$ is injective.

Proof. From Lemma 9(a) in [Varma 2014], we have $\phi_{n}^{\prime} * \phi_{n+1}^{\prime} * \phi_{n}^{\prime}(g)= \begin{cases}\lambda \cdot\left(\chi_{n}^{\prime}\right)^{-1}\left(h_{1} h_{2}\right) & \text { if } g=h_{1} y h_{2} \text { with } y \in \mathscr{Y}_{n}, h_{1}, h_{2} \in G_{n}^{\prime}, \\ 0 & \text { if } g \notin G_{n}^{\prime} \mathscr{Y}_{n} G_{n}^{\prime},\end{cases}$

where $\lambda=\operatorname{meas}\left(G_{n}^{\prime} \cap G_{n+1}^{\prime}\right)$ meas $\left(G_{n}^{\prime}\right)$. Now part 1 follows from Lemma 11 , as we have

$$
\tilde{\phi}_{n}^{\prime} * \tilde{\phi}_{n+1}^{\prime} * \tilde{\phi}_{n}^{\prime}=\left(\phi_{n}^{\prime} * \phi_{n+1}^{\prime} * \phi_{n}^{\prime}\right)^{\sim} .
$$

Now we prove part 2 . It is enough to say that $\pi\left(\tilde{\phi}_{n}^{\prime} * \tilde{\phi}_{n+1}^{\prime} * \tilde{\phi}_{n}^{\prime}\right)$ acts by a nonzero multiple of the identity on $W_{n}^{\prime}$. This implies that $I_{n+1, n}^{\prime} \circ I_{n, n+1}^{\prime}$ is a nonzero multiple of the identity on $W_{n}^{\prime}$. From part $1, \tilde{\phi}_{n}^{\prime} * \tilde{\phi}_{n+1}^{\prime} * \tilde{\phi}_{n}^{\prime}$ is a positive linear combination 
of functions $\tilde{\phi}_{n, y}^{\prime}: \gamma \mapsto \tilde{\phi}_{n}^{\prime}\left(\gamma y^{-1}\right)$, where $y \in G_{n+1} \cap G(Y)$ is fixed and $G(Y)$ is the centralizer of $Y$ in $G$. Then the lemma follows from the fact that $\pi(y)$ acts trivially on $W_{n}^{\prime}$ for large $n$, so that

$$
\left.\pi\left(\tilde{\phi}_{n, y}^{\prime}\right)\right|_{W_{n}^{\prime}}=\left.\pi\left(\tilde{\phi}_{n}^{\prime}\right) \pi(y)\right|_{W_{n}^{\prime}}=\left.\pi\left(\tilde{\phi}_{n}^{\prime}\right)\right|_{W_{n}^{\prime}} .
$$

Theorem 23. Let $(\pi, W)$ be an irreducible admissible genuine representation of $\tilde{G}$.

(1) The set of maximal elements in $\mathcal{N}_{\text {tr }}(\pi)$ coincides with the set of maximal elements in $\mathcal{N}_{\mathrm{Wh}}(\pi)$.

(2) Let 0 be a maximal element in $\mathcal{N}_{\text {tr }}(\pi)$. Then the coefficient $c_{\mathscr{O}}$ equals the dimension of the space of degenerate Whittaker forms with respect to any pair $(Y, \varphi)$ such that $Y \in \mathbb{O}$ is arbitrary and $\varphi: \mathbb{G}_{m} \rightarrow \boldsymbol{G}$ satisfies $\operatorname{Ad}(\varphi(s)) Y=s^{-2} Y$ for all $s \in E^{\times}$.

Proof. Let 0 be a maximal element in $\mathcal{N}_{\text {tr }}(\pi)$. Choose $(Y, \varphi)$ such that $Y \in \mathbb{O}$ and $\varphi: \mathbb{G}_{m} \rightarrow \boldsymbol{G}$ satisfies $\operatorname{Ad}(\varphi(s)) Y=s^{-2} Y$. Then, from Lemma 20, for large $n$ we have

$$
\operatorname{dim} W_{n}=c_{\odot} .
$$

Therefore $W_{n} \neq 0$ and $W_{n}^{\prime} \neq 0$ for large $n$. From Lemma 21, the map $j_{n}^{\prime}$ is injective and maps surjectively onto $\left(W / N_{\chi}^{\prime} W\right)^{\left(H_{0}, \tilde{\chi}\right)}$. But from the second part of Lemma 22 and (9), $I_{n, m}^{\prime}$ is injective for large $n$ and $m>n$, which implies that

$$
W_{n, \chi}^{\prime}=\bigcup_{m>n} \operatorname{ker}\left(I_{n, m}^{\prime}\right)=0 .
$$

Thus $\operatorname{dim} \mathscr{W}=\operatorname{dim} W_{n}^{\prime}=\operatorname{dim} W_{n}=c_{\mathscr{C}}$, which proves part 2 of the theorem. In particular, $\mathscr{W} \neq 0$ and hence $\mathbb{O} \in \mathcal{N}_{\mathrm{Wh}}(\pi)$. Now we claim that 0 is maximal in

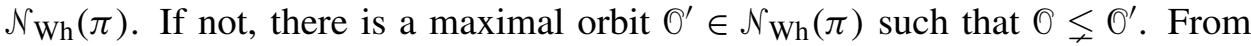
Proposition 19, there is $\mathbb{O}^{\prime \prime} \in \mathcal{N}_{\text {tr }}(\pi)$ such that $\mathbb{O}^{\prime} \leq \mathbb{O}^{\prime \prime}$. Therefore, $\mathcal{O} \subsetneq \mathbb{O}^{\prime \prime}$ and $\mathcal{O}, \mathcal{O}^{\prime \prime} \in \mathcal{N}_{\text {tr }}(\pi)$, a contradiction to the maximality of $\mathcal{O}$ in $\mathcal{N}_{\text {tr }}(\pi)$.

Let $\mathcal{O}$ be a maximal element in $\mathcal{N}_{\mathrm{Wh}}(\pi)$. From Proposition 19, there is an element in $\mathbb{O}^{\prime} \in \mathcal{N}_{\text {tr }}(\pi)$ such that $O \leq \mathcal{O}^{\prime}$. Take a maximal such $\mathcal{O}^{\prime}$. Then, by the result in the preceding paragraph, $\mathcal{O}^{\prime}$ is a maximal element in $\mathcal{N}_{\mathrm{Wh}}(\pi)$. But $\mathcal{O}$ is also maximal in $\mathcal{N}_{\text {Wh }}(\pi)$. Hence $\mathbb{O}=\mathcal{O}^{\prime}$. This proves that $\mathcal{O}$ is a maximal element in $\mathcal{N}_{\text {tr }}(\pi)$ too.

\section{Acknowledgements}

The author would like to express his gratitude to Professor D. Prasad and Professor Sandeep Varma for their help and numerous suggestions at various points. Without their help and continuous encouragement this paper would not have been possible. 


\section{References}

[Li 2012] W.-W. Li, "La formule des traces pour les revêtements de groupes réductifs connexes, II: Analyse harmonique locale”, Ann. Sci. Éc. Norm. Supér. (4) 45:5 (2012), 787-859. MR 3053009 Zbl 06155586

[Li 2014] W.-W. Li, "La formule des traces pour les revêtements de groupes réductifs connexes, I: Le développement géométrique fin”, J. Reine Angew. Math. 686 (2014), 37-109. MR 3176600 Zbl 06296344

[Mœglin and Waldspurger 1987] C. Mœglin and J.-L. Waldspurger, "Modèles de Whittaker dégénérés pour des groupes $p$-adiques", Math. Z. 196:3 (1987), 427-452. MR 89f:22024 Zbl 0612.22008

[Mœglin and Waldspurger 1995] C. Mœglin and J.-L. Waldspurger, "Appendix I: Lifting of unipotent subgroups into a central extension”, pp. 273-277 in Spectral decomposition and Eisenstein series: a paraphrase of the Scriptures, Cambridge Tracts in Mathematics 113, Cambridge University Press, 1995. MR 97d:11083 Zbl 0846.11032

[Mœglin et al. 1987] C. Mœglin, M.-F. Vignéras, and J.-L. Waldspurger, Correspondances de Howe sur un corps p-adique, Lecture Notes in Math. 1291, Springer, Berlin, 1987. MR 91f:11040 Zbl 0642.22002

[Prasad 1992] D. Prasad, "Invariant forms for representations of $\mathrm{GL}_{2}$ over a local field", Amer. J. Math. 114:6 (1992), 1317-1363. MR 93m:22011 Zbl 0780.22004

[Rodier 1975] F. Rodier, "Modèle de Whittaker et caractères de représentations", pp. 151-171 in Non-commutative harmonic analysis (Marseille-Luminy, 1974), edited by J. Carmona et al., Lecture Notes in Math. 466, Springer, Berlin, 1975. MR 52 \#14165 Zbl 0339.22014

[Varma 2014] S. Varma, "On a result of Moeglin and Waldspurger in residual characteristic 2", Math. Z. 277:3-4 (2014), 1027-1048. MR 3229979 Zbl 06323349

Received February 10, 2014. Revised April 13, 2014.

Shiv PRAKash PATEL

SCHOOL OF MATHEMATICS

TATA InSTITUTE OF FUNDAMENTAL RESEARCH

HOMI BHABHA ROAD

COlaba

Mumbai 400005

INDIA

shiv@math.tifr.res.in 


\title{
PACIFIC JOURNAL OF MATHEMATICS
}

\author{
msp.org/pjm
}

Founded in 1951 by E. F. Beckenbach (1906-1982) and F. Wolf (1904-1989)

\section{EDITORS}

Don Blasius (Managing Editor)

Department of Mathematics

University of California

Los Angeles, CA 90095-1555

blasius@math.ucla.edu

\author{
Paul Balmer \\ Department of Mathematics \\ University of California \\ Los Angeles, CA 90095-1555 \\ balmer@math.ucla.edu \\ Robert Finn \\ Department of Mathematics \\ Stanford University \\ Stanford, CA 94305-2125 \\ finn@math.stanford.edu \\ Sorin Popa \\ Department of Mathematics \\ University of California \\ Los Angeles, CA 90095-1555 \\ popa@math.ucla.edu
}

\author{
Vyjayanthi Chari \\ Department of Mathematics \\ University of California \\ Riverside, CA 92521-0135 \\ chari@math.ucr.edu \\ Kefeng Liu \\ Department of Mathematics \\ University of California \\ Los Angeles, CA 90095-1555 \\ liu@math.ucla.edu \\ Jie Qing \\ Department of Mathematics \\ University of California \\ Santa Cruz, CA 95064 \\ qing@ cats.ucsc.edu
}

\section{PRODUCTION}

Silvio Levy, Scientific Editor, production@msp.org

\section{SUPPORTING INSTITUTIONS}

ACADEMIA SINICA, TAIPEI

CALIFORNIA INST. OF TECHNOLOGY

INST. DE MATEMÁTICA PURA E APLICADA

KEIO UNIVERSITY

MATH. SCIENCES RESEARCH INSTITUTE

NEW MEXICO STATE UNIV.

OREGON STATE UNIV.

\author{
STANFORD UNIVERSITY \\ UNIV. OF BRITISH COLUMBIA \\ UNIV. OF CALIFORNIA, BERKELEY \\ UNIV. OF CALIFORNIA, DAVIS \\ UNIV. OF CALIFORNIA, LOS ANGELES \\ UNIV. OF CALIFORNIA, RIVERSIDE \\ UNIV. OF CALIFORNIA, SAN DIEGO \\ UNIV. OF CALIF., SANTA BARBARA
}

\author{
Daryl Cooper \\ Department of Mathematics \\ University of California \\ Santa Barbara, CA 93106-3080 \\ cooper@math.ucsb.edu \\ Jiang-Hua Lu \\ Department of Mathematics \\ The University of Hong Kong \\ Pokfulam Rd., Hong Kong \\ jhlu@maths.hku.hk \\ Paul Yang \\ Department of Mathematics \\ Princeton University \\ Princeton NJ 08544-1000 \\ yang@math.princeton.edu
}

These supporting institutions contribute to the cost of publication of this Journal, but they are not owners or publishers and have no responsibility for its contents or policies.

See inside back cover or msp.org/pjm for submission instructions.

The subscription price for 2015 is US \$420/year for the electronic version, and \$570/year for print and electronic.

Subscriptions, requests for back issues and changes of subscribers address should be sent to Pacific Journal of Mathematics, P.O. Box 4163, Berkeley, CA 94704-0163, U.S.A. The Pacific Journal of Mathematics is indexed by Mathematical Reviews, Zentralblatt MATH, PASCAL CNRS Index, Referativnyi Zhurnal, Current Mathematical Publications and Web of Knowledge (Science Citation Index).

The Pacific Journal of Mathematics (ISSN 0030-8730) at the University of California, c/o Department of Mathematics, 798 Evans Hall \#3840, Berkeley, CA 94720-3840, is published twelve times a year. Periodical rate postage paid at Berkeley, CA 94704, and additional mailing offices. POSTMASTER: send address changes to Pacific Journal of Mathematics, P.O. Box 4163, Berkeley, CA 94704-0163.

PJM peer review and production are managed by EditFLOW ${ }^{\circledR}$ from Mathematical Sciences Publishers.

\section{PUBLISHED BY}

\section{mathematical sciences publishers \\ nonprofit scientific publishing}

http://msp.org/

(C) 2015 Mathematical Sciences Publishers 


\section{PACIFIC JOURNAL OF MATHEMATICS}

Volume $273 \quad$ No. $1 \quad$ January 2015

Maximal estimates for Schrödinger equations with inverse-square potential 1

ChangXing MiaO, JUNYong Zhang and JiQiang Zheng

Vassiliev Invariants of Virtual Legendrian Knots

PATRICIA CAHN and ASA LEVI

Some results on the generic vanishing of Koszul cohomology via

deformation theory

JIE WANG

Conformal metrics with constant curvature one and finitely many conical

singularities on compact Riemann surfaces

QING Chen, WeI WANG, Yingyi Wu and BIN XU

$\mathbb{Q}$-bases of the Néron-Severi groups of certain elliptic surfaces

MASAMICHI KURODA

On a prime zeta function of a graph

TAKehiro Hasegawa and SEIKEN SAIto

On Whittaker modules for a Lie algebra arising from the 2-dimensional torus 147

SHaObin TAN, QING WANG and ChENGKANG XU

Fréchet quantum supergroups

AXEL DE GOURSAC

Generators of the Gauss-Picard modular group in three complex dimensions

BaoHua Xie, JieYan Wang and YuePing Jiang

Complete characterization of isolated homogeneous hypersurface singularities

STEPHEN YAu and HuAiQING ZuO

A theorem of Mœglin and Waldspurger for covering groups

SHIV PRAKASH PATEL

Spanning trees and random walks on weighted graphs 